\title{
THE PLACE OF HEALTH CARE IN SOCIAL SERVICES FOR PEOPLE WITH DISABILITIES
}

\author{
Daniela Konstantinova \\ Faculty of Public Health and Health Care, "Angel Kanchev" University of Ruse
}

\begin{abstract}
INTRODUCTION: Increased demand for services for long-term care for the elderly and disabled people is associated with the aging population in Bulgaria. In the methodologies of offering services attention is paid to the quality of social services and nutrition, whereas health care remains in the background. Consumers surveyed say that the quality of health care is at the first place.

AIM: To investigate the views of the users of social services and their relatives about the place of health care in meeting their needs.

MATERIALS AND METHODS: Study of regulatory documents - strategies, laws and methodological guidance related to the provision of social services for the elderly. A survey was prepared for the purpose of the study.

RESULTS AND DISCUSSION: We need better cooperation and coordination in the delivery of health and social services for the elderly and disabled, as well as an expansion of the circle of professionals providing longterm care for sick elderly people and people with disabilities. Regulation of professional responsibilities, individual specialists having the right to take a personal decision and introduction of missing professional units in our country will lead to better health care.
\end{abstract}

Keywords: elderly, social services in the community, health care

\section{INTRODUCTION}

The increased demand for services of long-term care for the elderly and disabled people is connected to the aging population in Bulgaria (1). In the methodologies of delivering services, special attention is paid to the quality of social services and nutrition, whereas health care remains in the background.

Bulgaria is among the countries where the risk of poverty or social exclusion of the population is above the average in the EU (2). Services of long-term care and other social and health services for elderly

Address for correspondence:

Daniela Kostadinova

8 Studentska Str

7004 Ruse

Bulgaria

e-mail: ddraganova@uni-ruse.bg and disabled people in Bulgaria are provided by two separate systems - social services system and health care system. Social services, defined as „activities that enhance and extend the capabilities of a person to lead an independent lifestyle, and are carried out in specialized institutions and in the community" are regulated by the Social Assistance Act (SAA) and the Regulations for Implementation of the social assistance (RISA) (1).

On the other hand, long-term healthcare services are regulated by the Law of Healthcare and the Hospitals Act (LHHA) and provided in different kinds of specialized medical institutions such as hospitals for further and continuous treatment, rehabilitation hospitals, long-term treatment and rehabilitation, state psychiatric hospitals and centers for mental health and hospices. 
The above-mentioned definitions of long-term care suggest encouragement and development of the interaction between social and health services.

\section{AIM}

To investigate the views of the users of social services and their relatives about the place of care in satisfying and meeting their needs.

For the purposes of the study legal documents were studied and users of social services and their families were interviewed.

\section{MATERIALS AND METHODS}

Study of regulatory documents, such as strategies, laws and methodological guidance related to the provision of social services for the elderly was performed. For the purpose of the study a survey identifying the needs of service users and their relatives was conducted.

The following methods were used:

- Documentary method (content analysis of documents) - used to examine the legal documents - strategies, laws and methodological guidance related to the provision of health and social services to people with disabilities; measures to improve the nation's health; structure and activities of social services in the community. The following documents were studied:

$\diamond$ Law on the professional organization of nurses, midwives and associated medical specialists;

$\diamond$ Development Strategy of Health Care in Bulgaria 2013 - 2020;

$\diamond$ Rules for the implementation of the Social Assistance Act;

$\diamond$ National Strategy for long-term care for the period 2014-2020.

- Sociological method is used to collect and analyze reliable information about the place of healthcare needs among the other needs of people with disabilities. A survey with 5 questions was conducted and 40 adults with disabilities and 40 of their relatives were interviewed.

- Statistical methods for processing and analysis of information - SPSS 16.0 was used.

The term "aging" is closely related to the concept of „life expectancy.” Thanks to scientific, technological and social progress and the advances of medicine, healthcare and many other factors, favorable conditions are set up for the increase of not only the individual, but also the average life expectancy in all developed countries (3).

Loss of physical strength, increasing pathology and high mortality rate are associated with ageing, determine the greater needs of older people and people with disabilities of medical care, social protection and assistance.

Different natural and social factors and conditions - material and cultural wealth, rational way of life, ecological environment, scientific advances of medical science and practical healthcare and others may have significant influence on the prolongation of human life, respectively on slowing the aging process (3).

Aging can pave the way for the emergence of diseases, and they in turn can distort the normal functioning of the body. Often occurring later in life, chronic diseases (atherosclerosis, tumors, metabolic diseases, diseases of the musculoskeletal system, etc.) are usually due to age-related changes. This determines the fear of death that is increasing with advancing age and illness and determines the high demand for health care.

Table 1 outlines the needs identified by relatives of adults with disabilities.

The relatives believe that in addition to health care, a major problem is the lack of free time. The same questions are answered by the consumers of social services. Their results are presented in Table 2.

For consumers, the most important ones are health care and the availability of nurses in the social institution which gives them peace of mind and security for their health. There was a difference of $15 \%$ in the responses of the second group, adults with dis-

Table 1. Need for care according to relatives of adults with disabilities

\begin{tabular}{lcc}
\hline Needs & Number & Percent \\
Social contacts & 3 & 7.5 \\
Health care & 16 & 40.0 \\
Free time & 9 & 22.5 \\
Culture & 3 & 7.5 \\
Nutrition & 2 & 5.0 \\
All of the above & 7 & 17.5 \\
& 40 & 100.0 \\
\hline
\end{tabular}


The Place of Health Care in Social Services for People with Disabilities

Table 2. Need for care according to consumers of social services - adults with disabilities

\begin{tabular}{lcc} 
Needs & Number & Percent \\
Social contacts & 3 & 7.5 \\
Health care & 22 & 55.0 \\
Free time & 7 & 17.5 \\
Culture & 2 & 5.0 \\
Nutrition & 1 & 2.5 \\
All of the above & 5 & 12.5 \\
& 40 & 100.0 \\
\hline \hline
\end{tabular}

abilities, when it comes to the importance given to health care.

Asked whether they believe it is enough to have a nurse and a social worker to care for them, 34 people (85\% of respondents) answer no, an equal number believe that a permanent presence of a doctor is not necessary. All agree that sufficient care could be provided by a nurse. According to the methodological guidelines, the provider of social services is obliged to have an employee with appropriate education, responsible for implementing the criteria and standards for health care without specifying his specialty (4).

According to data from the Information System for permanent disability of the population over 16 years of age of the National Center for Public Health and Analysis (NCPHA) based on annual processing of expert decisions issued by LEDC and NEPC after 2008, there was an increase in the number of persons with certain permanent disability for life, and in 2012 it reached 39,738 individuals (1).

The above-mentioned data and analysis of the situation clearly show that the increasingly aging population will also require increased public spending on long-term care as older people who have lost their autonomy and in need of intensive care, will constitute the fastest growing social group in society in the future. This will lead to an increase in the need for the development of health and social services and networks of solidarity and care (1).

\section{RESULTS AND DISCUSSION}

The different distribution of responsibilities and the different ways of organizing medical and social care and the differing legislation lead to the need for implementation of new models and their subse- quent regulation according to the specifics of our country (1).

We need better cooperation and coordination in the delivery of health and social services for the elderly and disabled, as well as expanding the circle of professionals providing long-term care for sick elderly people and people with disabilities (5). Regulation of professional responsibilities, the right of individual specialists to take a decision by themselves and the introduction of professional units missing in our country will lead to better care.

To improve the quality of care it is necessary to improve the equipment, structure and professional capacity of staff and to increase the monitoring of following the criteria and standards for the provision of health and social services.

\section{REFERENCES}

\section{NATIONAL STRATEGY FOR LONG TERM CARE 2014-2020}

2. National Social Report of the Republic of Bulgaria for 2013-2014 (adopted by the Council of Ministers of the Republic of Bulgaria on 04.30.2014)

3. Petrov P. Fundamentals of Geragogics. Sofia: Avangard Prima; 2005.

4. Methodical guidelines of the specialized institutions for social services and social services in the community in implementing the standards and criteria for health care, 2007

5. http://ec.europa.eu/health-eu/care_for_me/ long_term_care/index_bg.htm 